\title{
Pseudomonas syringae CC1557: A Highly Virulent Strain With an Unusually Small Type III Effector Repertoire That Includes a Novel Effector
}

\author{
Kevin L. Hockett, ${ }^{1}$ Marc T. Nishimura, ${ }^{2}$ Erick Karlsrud, ${ }^{1}$ Kevin Dougherty, ${ }^{1}$ and David A. Baltrus ${ }^{1}$ \\ ${ }^{1}$ School of Plant Sciences, University of Arizona, Tucson, AZ 85721-0036, U.S.A.; ${ }^{2}$ Department of Biology, University \\ of North Carolina, Chapel Hill, NC 27599, U.S.A.
}

Submitted 26 November 2013. Accepted 5 May 2014.

\begin{abstract}
Both type III effector proteins and nonribosomal peptide toxins play important roles for Pseudomonas syringae pathogenicity in host plants, but whether and how these pathways interact to promote infection remains unclear. Genomic evidence from one clade of $\boldsymbol{P}$. syringae suggests a tradeoff between the total number of type III effector proteins and presence of syringomycin, syringopeptin, and syringolin A toxins. Here, we report the complete genome sequence

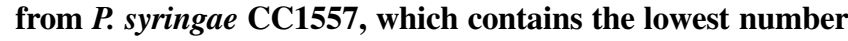
of known type III effectors to date and has also acquired genes similar to sequences encoding syringomycin pathways from other strains. We demonstrate that this strain is pathogenic on Nicotiana benthamiana and that both the type III secretion system and a new type III effector, hopBJ1, contribute to pathogenicity. We further demonstrate that activity of HopBJ1 is dependent on residues structurally similar to the catalytic site of Escherichia coli CNF1 toxin. Taken together, our results provide additional support for a negative correlation between type III effector repertoires and the potential to produce syringomycin-like toxins while also highlighting how genomic synteny and bioinformatics can be used to identify and characterize novel virulence proteins.
\end{abstract}

Pseudomonas syringae, a bacterial phytopathogen of many crop species, utilizes a diverse arsenal of virulence factors to infect host plants (Hirano and Upper 2000; O'Brien et al. 2011). Much of the previous research on pathogenicity in $P$. syringae has focused on identifying and characterizing virulence factors within strains and on categorizing their presence across the species (Baltrus et al. 2011; Hwang et al. 2005; Lindeberg et al. 2012; O'Brien et al. 2011). Although this accumulation of knowledge has enabled tests of protein function during infection, many questions remain as to how virulence pathways interact and evolve at a systems level (Baltrus et al. 2011; Cunnac et al. 2011; Hwang et al. 2005). In the absence of direct tests, identification of similar genomic trends across independent

\section{K. L. Hockett and M. T. Nishimura contributed equally to this work.}

Corresponding author: D. A. Baltrus; Telephone: +1.520 .626 .8215 ; Fax: +1.520.621.7186; E-mail: Baltrus@email.arizona.edu

* The $\boldsymbol{e}$-Xtra logo stands for "electronic extra" and indicates that four supplementary figures are published online.

(C) 2014 The American Phytopathological Society lineages provides strong support for the presence of such interactions over evolutionary time scales.

One of the main contributors to pathogenicity within $P$. syringae is a type III secretion system (T3SS) (O'Brien et al. 2011). The T3SS encodes a structure that translocates bacterial effector proteins (type III effectors [T3E]) into host cells to disrupt host physiological pathways and enable successful infection (Lindeberg et al. 2012). P. syringae genomes typically contain approximately 10 to 40 total T3E, the exact actions of which depend on the totality of effector repertoires as well as host genotype (Baltrus et al. 2011; Lindeberg et al. 2012; O'Brien et al. 2011). Another main contributor to pathogenicity within many $P$. syringae strains is nonribosomal peptide (NRP) toxin pathways. NRP pathways encode proteins that act as an assembly line, elongating and decorating peptide chains involved in pathogenicity (Bender et al. 1999). NRP pathways are often not conserved between closely related strains but, even if present, can differ in their outputs based on nucleotide polymorphisms (Baltrus et al. 2011; Bender et al. 1999; Hwang et al. 2005).

Multiple reports have suggested functional overlap or phenotypic interactions between T3E and NRP pathways. For instance, virulence functions of the toxin coronatine can be partially restored by the toxin syringolin A as well as T3E HopZ1 and AvrB1 (Cui et al. 2010; Jiang et al. 2013; Melotto et al. 2006; Schellenberg et al. 2010). Furthermore, within multilocus sequence analysis (MLSA) group II $P$. syringae strains as defined by Sarkar and Guttman (2004), there exists a negative correlation between presence of conserved toxin pathways (syringolin A, syringopeptin, and syringomycin) and size of the T3E repertoire compared with other phylogenetic clades (Baltrus et al. 2011) (Supplementary Fig. S1). In fact, MLSA group II strains possess the lowest number of T3E among analyzed genomes across the species. This correlation is strengthened by the observation that strains from pv. pisi possess some of the largest T3E repertoires within MLSA group II and have lost all three toxin pathways (Baltrus et al. 2013). Whether this negative correlation between NRP and T3E reflects an unrecognized difference in disease ecology is yet to be determined, although strains from MLSA group II are thought to survive as epiphytes much better than other clades within $P$. syringae (Feil et al. 2005).

The hypothesis of a phenotypic tradeoff between T3E and NRP pathways would be bolstered by discovery of an independent clade of $P$. syringae that has gained toxins similar to those of the MLSA group II strains but which also possesses a reduced effector repertoire. We have analyzed the genomes of a small clade of $P$. syringae isolated from environmental 
sources (Baltrus et al. 2014; Morris et al. 2008) and have found independent evidence supporting this genomic trend. Specifically, compared with a closely related outgroup, genomes for two strains appear to have lost $\mathrm{T} 3 \mathrm{E}$ while gaining a path-

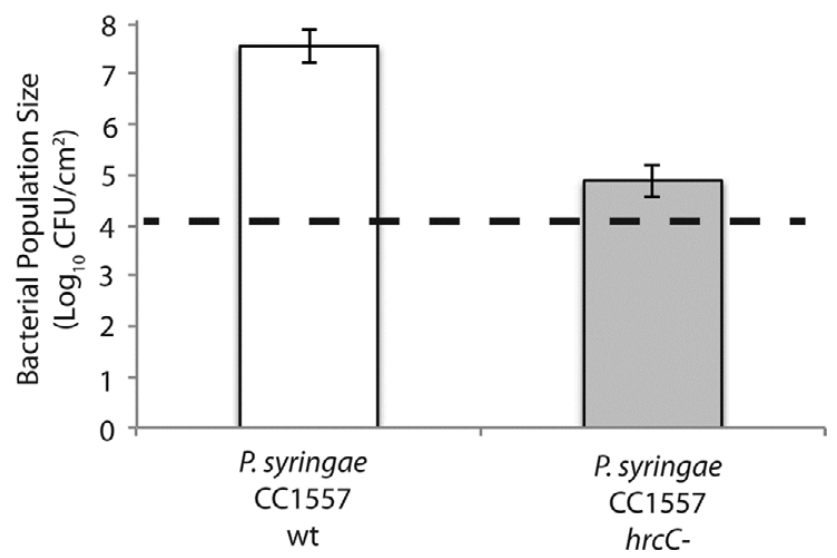

Fig. 1. Pseudomonas syringae $\mathrm{CC} 1557$ virulence in Nicotiana benthamiana is type III-secretion dependent. Growth of wild-type $P$. syringae $\mathrm{CC} 1557$ as well as an $h r c C$ mutant was measured in planta 3 days postsyringe inoculation. Measurements are based on three independent experiments with at least four replicates in each. Bacterial population sizes are significantly different (Tukey's honestly significant difference, $P<0.05$ ) between strains. Error bars represent two standard errors. Dashed lines indicate approximate population sizes at day 0 , which were not significantly different. way which potentially encodes proteins for the production of a syringomycin-like toxin. We demonstrate that one of these strains, CC1557, can infect Nicotiana benthamiana and cause disease in a T3SS-dependent manner. We further show that virulence of this strain is significantly increased by the presence of one new T3E effector, HopBJ1, which shares similar structure and catalytic residues with the CNF1 from Escherichia coli. Therefore, independent evidence suggests that acquisition of NRP pathways correlates strongly with the loss of T3E families and further strengthens the idea of an ecological or evolutionary tradeoff between these virulence factors.

\section{RESULTS}

A complete genome sequence for $P$. syringae $\mathrm{CC1557.}$

2008). This genome consists of a 5,728,024-bp chromosome and a 53,629-bp plasmid (GenBank accession number AVEH02000000). According to PGAAP annotation (Angiuoli et al. 2008), this chromosome contains 5,001 open reading frames (ORF) while the single plasmid contains an additional 67 ORF.

\section{$P$ syringae $\mathrm{CC} 1557$ can infect and cause disease in $N$. benthamiana.}

$P$. syringae CC1557 was originally isolated from snow, whereas the closely related strain CC1466 was originally isolated from asymptomatic Dodecantheon pulchellum, a perennial herb (Morris et al. 2008). Using syringe inoculation under standard conditions, we have demonstrated that $P$. syringae

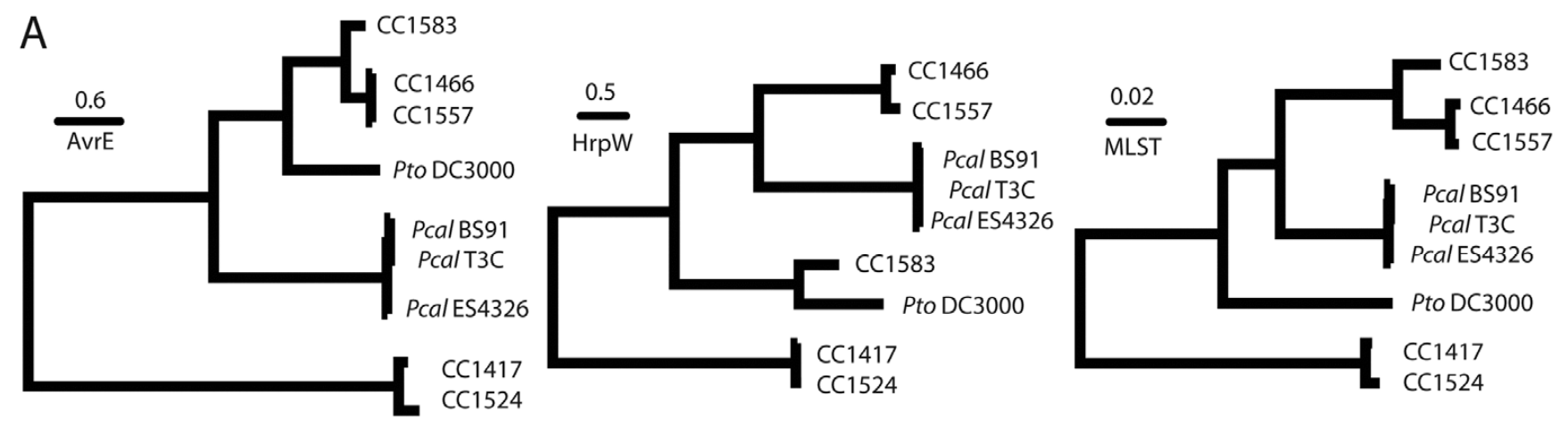

B

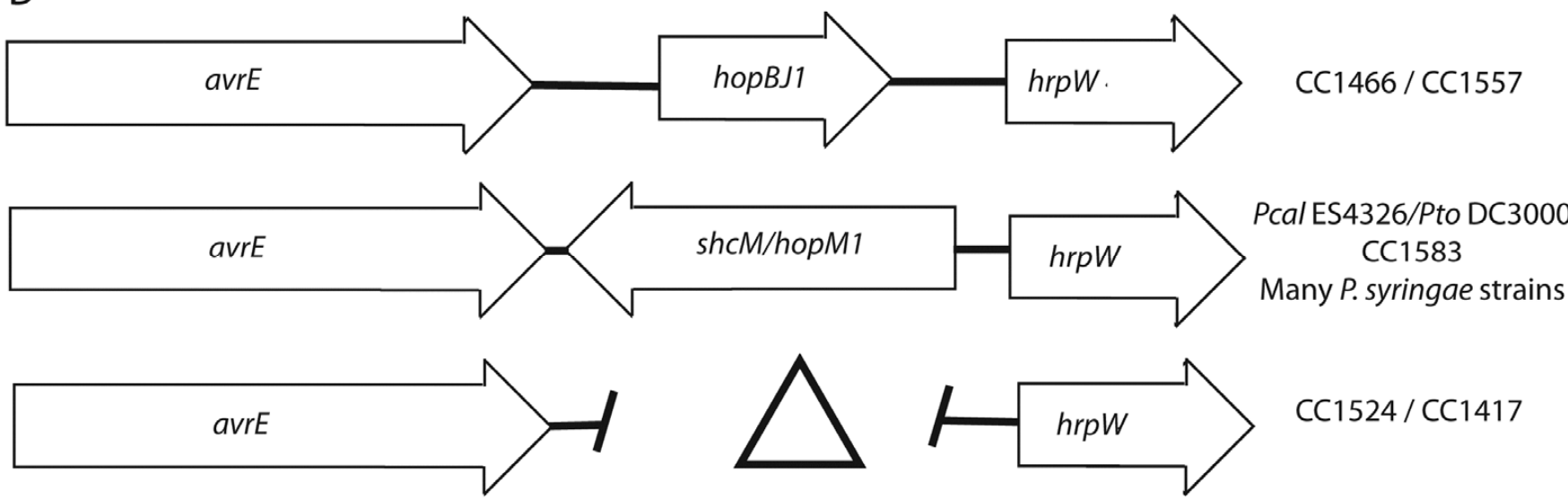

Fig. 2. Diversity of the CEL locus across Pseudomonas syringae sensu lato. A, Bayesian phylogenies for AvrE (left), HrpW (middle), and concatenated multilocus sequence analysis (MLSA) loci (right), for a subset of $P$. syringae strains. Posterior probabilities for all nodes are $>0.95$. Scale bars indicate number of amino acid changes. Phylogenetic patterns for both of these loci approximate relationships based on core genomes and MLSA loci, except that HrpW from $P$. syringae CC1583 clusters more closely with $P$. syringae pv. tomato DC 3000 . This suggests a recombination event at this locus within $P$. syringae CC1583. B, Genomic context of the CEL across $P$. syringae sensu lato. In most strains, hopM1 is bordered by avrE and hrpW. Some $P$. viridiflava strains lack hopM1 (Bartoli et al. 2014), while CC1557 and CC1466 have replaced this region with hopBJ1. Arrows in represent open reading frames while the gap and triangle represent a loss of this region within strains CC1524 and CC1417. 
CC1557 can grow to high population sizes in the apoplast of $N$. benthamiana after 3 days of infection (Fig. 1). This rate of growth is similar to the well-established pathogenic strain for N. benthamiana, P. syringae pv. syringae B728a (Supplementary Fig. S2). Furthermore, these large bacterial population sizes cause disease symptoms as shown by the visibility of tissue collapse (Fig. 2). High levels of growth and tissue collapse are both eliminated by deleting the $h r c C$ gene (Figs. 1 and 2), which encodes a main structural protein for the type III secretion pilus. Therefore, $\mathrm{CC} 1557$ virulence in $N$. benthamiana under laboratory conditions is T3SS dependent.

\section{The genomes of $P$. syringae $\mathrm{CC1557}$ and $\mathrm{CC} 1466$ encode a reduced $\mathrm{T3E}$ repertoire.}

We used bioinformatic methods as per Baltrus and associates (2011) to search the complete and draft genomes of $P$. syringae strains $\mathrm{CC} 1557$ and $\mathrm{CC} 1466$, and the closely related strain CC1583, for known T3E families (Baltrus et al. 2014). We found a total of four T3E shared across these three genomes, with eight T3E found only within CC1583 (Table 1). Therefore, together with HopBJ1 (see below), the genomes of CC1466 and CC1557 appear to encode a total of five potential T3E from known protein families. This stands in contrast to the immediate outgroup strain $\mathrm{CC} 1583$, which encodes a total of 12 potential T3E from known protein families.

\section{P. syringae CC1466 and CC1557 have horizontally acquired pathways for production of NRP that resemble syringopeptin and syringomycin.}

We used tBLASTn searches of the complete genome sequence of CC1557 in order to identify potential NRP toxin pathways, as per Baltrus and associates (2011). This genome contains highly similar full-length tBLASTn matches to a variety of proteins involved in syringomycin synthesis: SyrB (93\% protein sequence identity), SyrC (99\%), and SyrP (93\%) (data not shown). Further investigation of all complete genomes for $P$. syringae demonstrates that the region containing putative NRP-related loci in CC1557 is found in approximately the same genomic context on the chromosome as the syringomycinsyringopeptin pathways in $P$. syringae pv. syringae $\mathrm{B} 728 \mathrm{a}$ (Supplementary Fig. S3). Moreover, the genomic context surrounding these toxin pathways is conserved throughout $P$. syringae. Although regions of high similarity to the syringopeptin pathway do not appear to be present within CC1557, analysis of gene annotations suggests that a second NRP pathway occurs immediately downstream of the syringomycin-like region on the chromosome of $\mathrm{CC} 1557$ (data not shown). Although it is difficult to identify the NRP product of these CC1557 pathways by sequence alone, draft genomes sequences

Table 1. Type III effector family distribution ${ }^{\mathrm{a}}$

\begin{tabular}{|c|c|c|c|}
\hline Effector name & CC1557 & CC1466 & CC1583 \\
\hline avrE & $\mathrm{P}$ & $\mathrm{P}$ & $\mathrm{P}$ \\
\hline hopF2-1 & - & - & $\mathrm{P}$ \\
\hline hopF2-2 & - & - & $\mathrm{P}$ \\
\hline hopM1 & - & - & $\mathrm{P}$ \\
\hline hopY1 & $\mathrm{P}$ & $\mathrm{P}$ & $\mathrm{P}$ \\
\hline hорАA1 & - & - & $\mathrm{P}$ \\
\hline hopAG1 & - & - & $\mathrm{P}$ \\
\hline hорАН1 & - & - & $\mathrm{P}$ \\
\hline hорАН 2 & $\mathrm{P}$ & $\mathrm{P}$ & $\mathrm{P}$ \\
\hline hopAII & - & - & $\mathrm{P}$ \\
\hline hopAS1 & - & - & $\mathrm{P}$ \\
\hline hopBF1 & $\mathrm{P}$ & $\mathrm{P}$ & $\mathrm{P}$ \\
\hline hopBJ1 & $\mathrm{P}$ & $\mathrm{P}$ & - \\
\hline
\end{tabular}

${ }^{a}$ The letter $\mathrm{P}$ denotes presence of this particular effector family, whereas the symbol - denotes absence. suggest that this region is present in CC1466 but not CC1583. The most parsimonious explanation for this phylogenetic signal is that an immediate ancestor of CC1466 and CC1557 acquired this NRP region by horizontal transfer after divergence from the CC1583 lineage.

\section{A new T3E protein family is present}

in the genomes of both $P$. syringae CC1466 and CC1557.

Similarity searches of the $P$. syringae genomes demonstrated that hopM1, which encodes an effector protein largely conserved throughout $P$. syringae, had likely been lost within strains CC1466 and CC1557. Further investigation demonstrated that an ORF encoding a hypothetical protein had replaced hopM1 within these genomes (Fig. 2). In order to test whether this unknown protein is translocated into plant cells, we cloned the region encoding the predicted ORF into the translocation test vector pBAV178 and tested for delivery the C-terminal fragment of AvrRpt 2 as a fusion protein. We found that the resulting HopBJ1-AvrRpt2 fusion protein triggered cell death in both Arabidopsis thaliana Col-0 wild type and rps 2 lines, indicating that HopBJ1 itself was capable of triggering cell death in the absence of recognition of AvrRpt2 (Table 2; Fig. 3B). In order to verify that HopBJ1 triggered cell death without AvrRpt2, we delivered HopBJ1:HA into a variety of plant genotypes using P. syringae pv. tomato DC3000 D28E (Cunnac et al. 2011). Delivery of HopBJ1:HA can cause tissue collapse in a variety of Arabidopsis accessions (Table 2). All genotypes tested displayed strong cell death by either 24 or 48 h postinoculation.

HopBJ1-induced cell death is not dependent on the known resistance $(R)$-gene pathways because tissue collapse still occurs in eds 1 , pad4, or rarl mutants (Table 2). HopBJ1-induced cell death also occurs in plants expressing the salicylic aciddegrading enzyme NahG (Table 2). However, this rapid tissue collapse fails to occur out of a $h r c C$ - background of $P$. syringae pv. tomato DC3000, indicating that HopBJ1 secretion is dependent on a functioning T3SS (data not shown). The genomic region immediately upstream of hopBJl possesses a

Table 2. Responses of Arabidopsis accessions to HopBJ $1^{\mathrm{a}}$

\begin{tabular}{|c|c|}
\hline Arabidopsis accession & Reaction \\
\hline Col & ++ \\
\hline Col rps 2 & ++ \\
\hline Ws & ++ \\
\hline Ws eds1 & ++ \\
\hline Ws pad4 & ++ \\
\hline Ws rarl & ++ \\
\hline Ws $N a h G$ & ++ \\
\hline $\mathrm{Ag}-0$ & ++ \\
\hline Bur-0 & + \\
\hline Can-0 & + \\
\hline $\mathrm{Ct}-1$ & ++ \\
\hline Edi-0 & ++ \\
\hline $\mathrm{Hi}-0$ & + \\
\hline $\mathrm{Kn}-0$ & + \\
\hline Ler-0 & ++ \\
\hline Mt-0 & ++ \\
\hline No-0 & ++ \\
\hline Oy-0 & ++ \\
\hline Po-0 & ++ \\
\hline Rsch & ++ \\
\hline Sf-0 & + \\
\hline Tsu-0 & + \\
\hline Wil-2 & + \\
\hline Ws-0 & + \\
\hline Wu-0 & + \\
\hline $\mathrm{Zu}-0$ & + \\
\hline
\end{tabular}

${ }^{a}$ Gene names next to accession indicate mutant lines; ++ and + indicate hypersensitive response in 24 and $48 \mathrm{~h}$, respectively. 

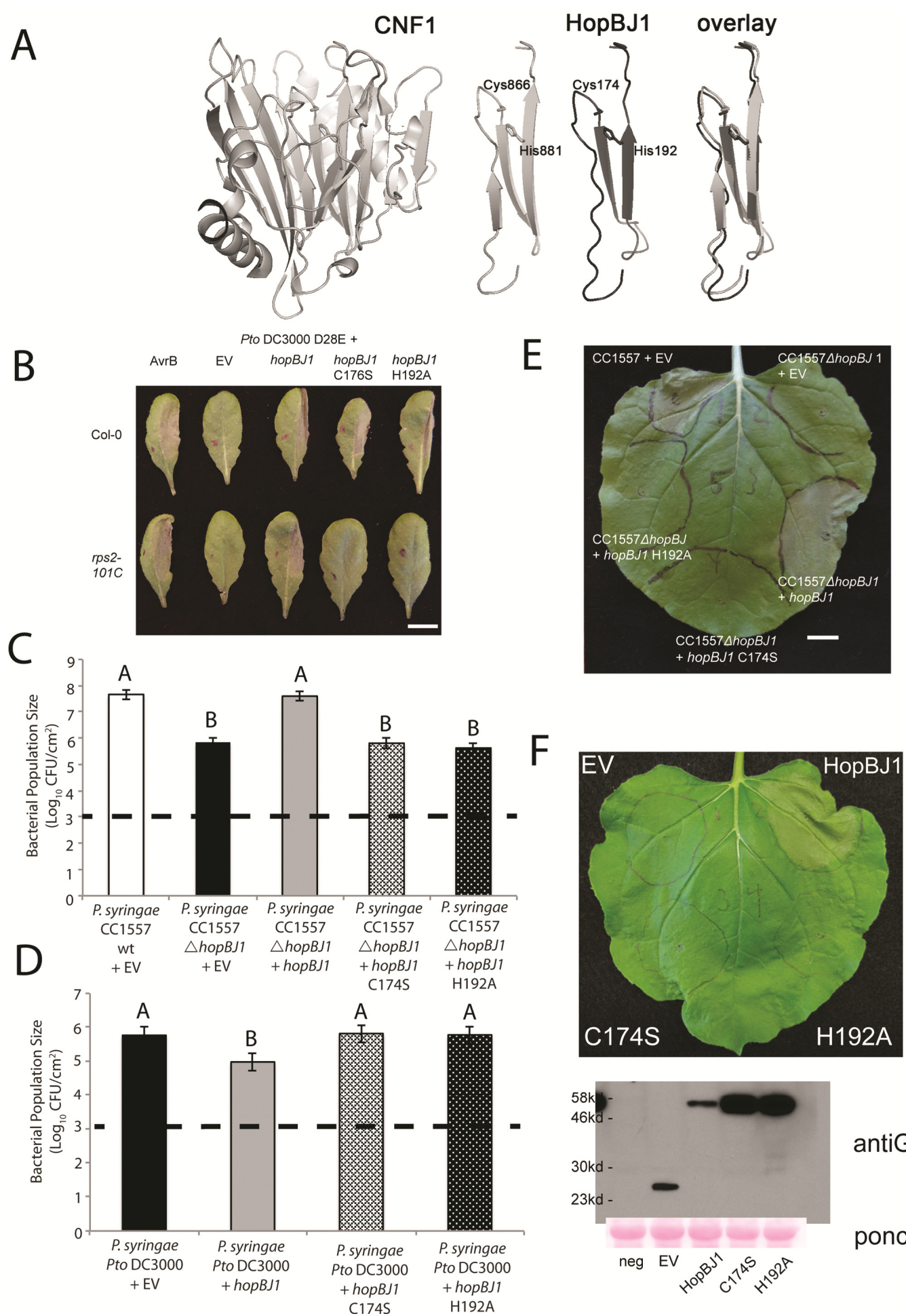

antiGFP

ponceau

Fig. 3. Protein motifs and catalytic residues within HopBJ1 are similar to the Escherichia coli CNF1 toxin. A, Despite no sequence similarity between HopBJ1 and CNF1 by BLASTp searches, modeling of tertiary protein structure demonstrates limited similarity in protein structure between the two. Importantly, the catalytic dyad of E. coli CNF1 (Cys866-His881) perfectly lines up with a putative catalytic dyad within HopBJ1 (Cys174 and His192). B, All constructs of HopBJ1 can be translocated by P. syringae pv. tomato DC3000 D28E (Cunnac et al. 2011) into Arabidopsis. Top: translocation tests into accession Col-0. Bottom: translocation tests into rps2-101C mutant plants. Translocation of AvrB is shown as a positive control and causes hypersensitive response in both plant backgrounds, while the DC3000 D28E mutant causes no cell death. HopBJ1 causes rapid cell death across both plant backgrounds when translationally fused to the C-terminus of AvrRpt2, whereas putative catalytic mutants only show cell death in Col- 0 . Picture is representative of 9 to 12 independent leaves per strain-plant combination C, Putative catalytic mutants of HopBJ1 cannot complement the growth defect of a CC1557 hopBJ1 deletion strain. D, Translocation of HopBJ1 but not either of the two putative catalytic mutants lowers growth of DC3000 on Arabidopsis Col-0. E, Wild-type and the complemented $\triangle h o p B J I$ deletion of strain CC1557 cause tissue collapse in Nicotiana benthamiana. Neither of the putative catalytic mutants complements the loss of virulence of the $\triangle h o p B J 1$ deletion strain. Picture is representative of multiple assays. F, Transient expression of HopBJ1 but not the putative catalytic mutants by Agrobacterium spp. in N. benthamiana causes cell death. Western blots and Ponceau staining for each of these Agrobacterium infiltrations are displayed below. Picture is representative of multiple assays. Each growth curve experiment consisted of at least two independent trials but representative data from only one trial is shown in each case. Letters indicate significant differences between bacterial population sizes between strains $(P<0.05$, Tukey's honestly significant difference). Dashed lines indicate approximate population sizes at day 0 , which were not significantly different. 
canonical hrp-box sequence (Supplementary Fig. S4) and, therefore, regulation of this gene likely takes place through the action of the $\sigma$ factor HrpL (Mucyn et al. 2014). Therefore, we conclude that this novel ORF encodes a new putative T3E family, HopBJ, and that this family possesses broad cytotoxicity. Further studies in diverse plant hosts will be required to determine the full extent of HopBJ cytotoxic activity.

\section{Deletion of HopBJ1 from $P$. syringae CC1557 causes \\ loss of disease symptoms and lowers bacterial populations in planta.}

We have created a deletion mutant of hopBJl in $P$. syringae strain $\mathrm{CC} 1557$ and have performed growth curves in planta using syringe infiltration. Deletion of hopBJl causes a repeatable loss of growth in planta after 2 days of infection (Fig. 4A). We further find that deletion of hopBJI leads to a loss of the tissue collapse phenotype after 3 days (Fig. 4B). We have been able to complement both of these phenotypes in planta through expression of hopBJl using its native promoter in trans (Fig. 4).

\section{HopBJ1 resembles cytotoxic genes present within other species.}

Currently, the closest BLASTp hits for HopBJ1 are hypothetical proteins from Serratia marcescens (34\% identity, YP_007346200.1) and Hahella chejuensis (30\% identity, YP_435189.1). However, modeling of protein structure using the Phyre2 web server demonstrates that HopBJ1 displays limited similarity to the E. coli CNF1 toxin (Fig. 3A). Surprisingly, amino acids critical for the deamidase functions of CNF1 appear to be conserved in HopBJ1 (Fig. 3A) as well as in similar proteins from $S$. marcescens and $H$. chejuensis (Buetow et al. 2001) (data not shown). This modeling of protein structure further suggests that $\mathrm{C} 174$ and $\mathrm{H} 192$ are a functional catalytic dyad within HopBJ1.

\section{Predicted catalytic residues of HopBJ1 are required for effector function.}

To test for functionality of predicted catalytic residues for HopBJ1, we measured the effect of putative catalytic dead mu- tants (C174S and H192A) in planta. Mutant versions of HopBJ1 are successfully translocated by strain DC3000 D28E into Arabidopsis, triggering RPS2-dependant cell death in Col0 . The wild-type HopBJ1:AvrRpt2 fusion also causes tissue collapse in rps2-101c plants, consistent with the ability of HopBJ1:HA to trigger cell death. (Fig. 3B). Moreover, both catalytic dead mutants are unable to complement virulence defects of a hopBJI deletion in CC1557 in N. benthamiana (Fig. $3 \mathrm{C}$ and $\mathrm{E})$. Both mutants, unlike the wild-type version, also fail to lower the growth of DC3000 in Arabidopsis (Fig. 3D) and fail to cause tissue collapse when transiently expressed in $N$. benthamiana (Fig. 3F). Therefore, as with similar residues in E. coli CNF1 (Buetow et al. 2001), we conclude that both $\mathrm{C} 174$ and $\mathrm{H} 192$ are required for the function of HopBJ1.

\section{DISCUSSION}

Recent advances in genome sequencing have spurred great interest in conservation and diversification of virulence pathways across $P$. syringae (O'Brien et al. 2011; Studholme 2011). Although genome gazing often leads to the identification of trends across a species, single observations must be treated with skepticism in the absence of additional data. One particularly interesting yet relatively little understood pattern across genomes involves a negative correlation in MLSA group II between size of T3E repertoire and presence of NRP pathways (Baltrus et al. 2011). Importantly, this trend does not appear to be a result of sampling bias or failure to discover novel T3E because strains within this clade have been thoroughly screened at both the genetic and transcriptome level (Baltrus et al. 2011; Mucyn et al. 2014). Indeed, the entire $h r p L$ regulon, the major transcriptional regulator for all $P$. syringae T3E, is reduced within MLSA group II compared with other clades (Mucyn et al. 2014).

Strains CC1466 and CC1557 diverge early in the phylogeny of $P$. syringae sensu lato, and have not been isolated from diseased plants in nature (Morris et al. 2008). We have shown that CC1557 can act as a pathogen of $N$. benthamiana and, as with all other phytopathogenic $P$. syringae strains, that virulence
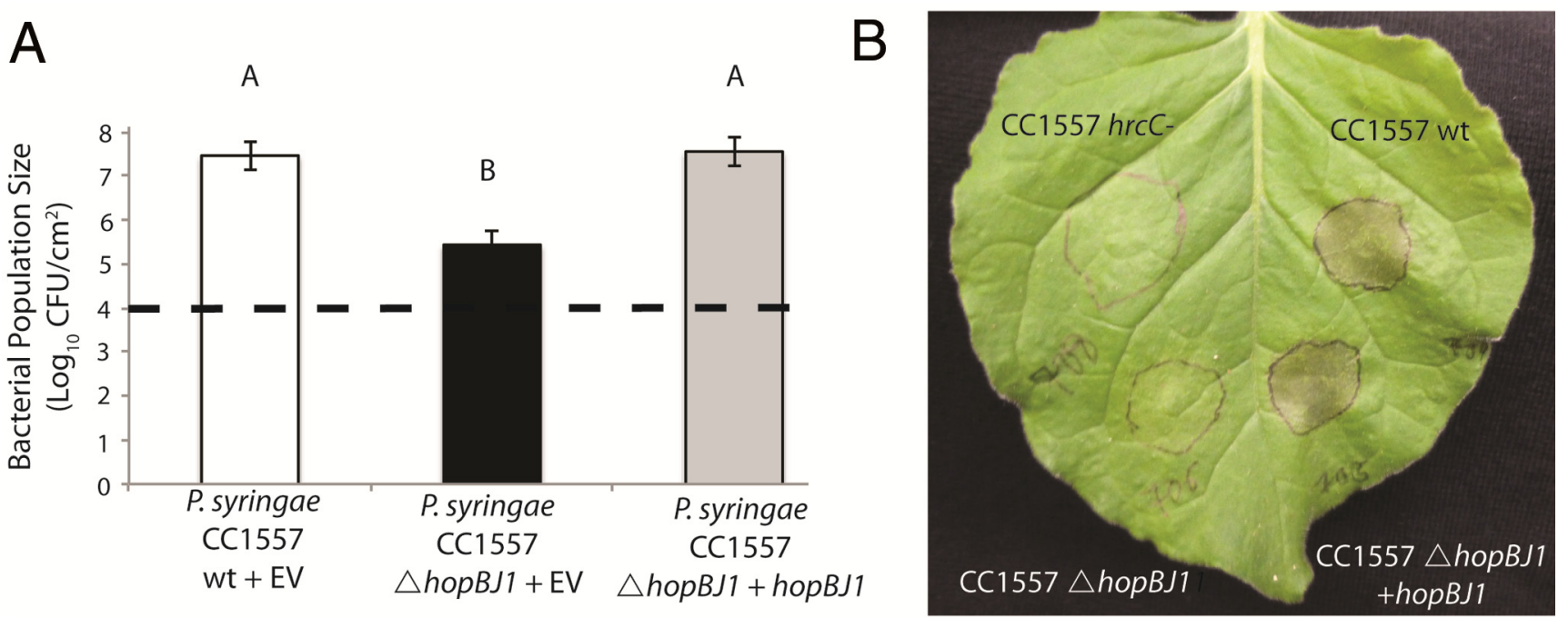

Fig. 4. HopBJ1 is a virulence factor for Pseudomonas syringae CC1557 in Nicotiana benthamiana. A, We created a deletion mutant of hopBJ1 in P. syringae $\mathrm{CC} 1557$, and complemented this strain by expressing hopBJ1 from a plasmid using the native promoter. Deletion of hopBJ1 lowers growth of $P$. syringae CC1557 3 days postinoculation. Both of these phenotypes are complemented by expression of wild-type hopBJ1 in trans. Three independent experiments were performed with at least four replicates in each treatment. Letters represent statistical differences within an analysis of variance (Tukey's honestly significant difference, $P<0.05)$. Error bars represent two standard errors. Dashed lines indicate approximate population sizes at day 0 , which were not significantly different. B, Leaves from 2-week-old $N$. Benthamiana plants were syringe inoculated with various strains derived from $P$. syringae CC1557. Three days after inoculation, plants showed evidence of tissue collapse dependent on a functioning type III secretion system (compare wild-type versus $h r c C$ mutant) as well as HopBJ1 (compare hopBJ1 deletion strain versus hopBJ1 complement). Picture is representative of multiple trials. 
requires a functional T3SS (Fig. 1). We have found that both of these genomes contain few T3E, with only five loci known to be present and shared by both (Table 1). Genomic comparisons between these two strains have further demonstrated that both contain an NRP toxin pathway very similar to those encoding syringomycin. As is the nature of NRP toxins, even a single amino acid change can have dramatic consequences on the final toxin product chemistry (Bender et al. 1999); however, it is important to note that many of the predicted ORF in these regions are highly similar ( $>90 \%$ protein identification) to syringomycin ORF present within MLSA group II strains. Comparison with closely related outgroup strains demonstrates that acquisition of this NRP pathway is evolutionarily correlated with loss of $\mathrm{T} 3 \mathrm{E}$ families. Indeed, the most closely related sequenced outgroup strain, CC1583, lacks this NRP pathway and contains twice the number of known T3E loci (Table 1). As such, we interpret these patterns as independent evidence suggesting an evolutionary or ecological tradeoff between presence of syringomycin and number of T3E within the strain. We also note that there appears to be an additional, uncharacterized NRP pathway downstream of the syringomycinlike region within CC1557 (data not shown). Directed experiments will need to be performed to assess the contribution of these NRP pathways to virulence.

What selective pressure could underlie such a negative correlation between $\mathrm{T} 3 \mathrm{E}$ and NRP toxins? It is possible that the functions of these NRP toxins are redundant with a specific suite of T3E. In this case, T3E loss can take place because there is no longer positive selection to counter selection against evolutionary (such as recognition by the plant immune system) or physiological costs. For MLSA clade II and CC1557, acquisition of syringomycin and, potentially, additional NRP pathways could render the functions of some T3E obsolete. If this is the case, we further predict that the potential second NRP toxin pathway (downstream of the syringomycin-like pathway in CC1557) could have functions similar to those of syringopeptin. It is also possible that disease ecologies of most MLSA group II strains, as well as CC1466 and CC1557, differ compared with other focal $P$. syringae strains. We have a rudimentary understanding of differences in disease ecology throughout $P$. syringae pathogens, although it is recognized that differences do exist across the phylogeny (Clarke et al. 2010). Specifically, one clade of strains from MLSA group II has exchanged the canonical $P$. syringae T3SS with a second divergent T3SS at an independent genomic location while still maintaining toxin pathways. Although these strains can grow in planta, it is unknown whether they can cause disease (Clarke et al. 2010). Furthermore, infection of woody hosts has arisen independent times on multiple hosts yet such strains are still dependent on a functioning T3SS (O'Brien et al. 2011; Ramos et al. 2012). Strains that contain syringopeptin and syringomycin potentially cause disease on a wider host range than other groups, which may be enabled by the generality of NRP toxins (Baltrus et al. 2011; Hwang et al. 2005; Quigley and Gross 1994). Alternatively, these strains may survive and persist across a variety of environments and host plants but only rarely reach sufficiently high population densities to cause disease. MLSA group II strains appear to survive better as epiphytes compared with other groups, and the presence of NRP toxins may tie into this strategy (Feil et al. 2005). Moreover, such differences in disease ecology may be difficult to precisely measure because they may only be visible under natural conditions of infection and dispersal.

It is possible that there are a number of undescribed $\mathrm{T} 3 \mathrm{E}$ families within CC1466 and CC1557 and, to this point, we have successfully used comparisons of genome synteny to identify HopBJ1. hopBJl is present within the conserved effector locus within these strains and, as such, is proximate to both the structural genes for the T3SS and the T3E avrE. Although it is difficult to precisely map out the evolutionary history of this region, it does appear that hopBJl has been recombined into this locus in place of hopMl (Fig. 4). In some respects, recombination of hopM1 from this locus is analogous to a situation witnessed in MLSA group I $P$. syringae strains, where hopMl alleles have been cleanly swapped through recombination (Baltrus et al. 2011). HopM1 is an effector whose presence (although not necessarily in functional form) is conserved throughout $P$. syringae sensu stricto, and has been shown to act redundantly with AvrE during infection of plant hosts (Badel et al. 2006; Kvitko et al. 2009). Moreover, in contrast to frameshift or nonsense mutations disrupting the coding sequence of hopMl in $P$. syringae, presence of this gene is polymorphic within $P$. viridiflava. Although some strains maintain an intact version, others lack hopMl completely (Araki et al. 2006; Bartoli et al. 2014). It is currently unknown whether HopBJ1 can act redundantly with AvrE on specific hosts, although the fact that our single deletion mutant shows a virulence phenotype on $N$. benthamiana speaks against this possibility. Moreover, because HopBJ1 appears to be responsible for a significant portion the growth of CC1557 in planta, it will be interesting to see how this effector behaves within different strain backgrounds on different hosts.

HopBJ1 itself displays protein structure similarity to CNF1 toxin found within pathogenic $E$. coli strains. CNF1 functions by causing deamidation of a glutamine residue, crucial for GTP hydrolysis for small GTPases of the Rho family, thereby leading to constitutive activation and actin disruption (Lemonnier et al. 2007). Furthermore, changing either of these amino acids within HopBJ1 eliminates virulence activity of CC1557 in planta, as well as during transient expression by Agrobacterium spp., and eliminates avirulence activity when delivered from DC3000 in Arabidopsis (Fig. 3). Potential functional parallels between CNF1 and HopBJ1 are particularly interesting because Rho GTPases are known to regulate cytoskeletal dynamics in plants (Mucha et al. 2011), actin has a demonstrated role in basal defense (Henty-Ridilla et al. 2013), and the $P$. syringae effector HopZ1a has been shown to target tubulin in order to promote virulence (Lee et al. 2012). If HopBJ1 does, indeed, target Rho family GTPases to disrupt the cytoskeleton, it would represent a striking example of molecular convergence across plant and animal pathogens.

The pathology of HopBJ1 on Arabidopsis, when delivered from strain DC3000, suggests that HopBJ1 could be acting as a general toxin because cell death appears regardless of accession and is not dependent on typical $R$-gene-related host defense pathways (Table 2). Moreover, HopBJ1 does act as an avirulence factor when delivered from DC3000 because cell death limits the growth of this strain in Arabidopsis (Fig. 3D). In some ways, this may be similar to the functions of AvrE, which was identified as causing cell death in soybean (Kobayashi et al. 1989). It is tempting to think that both AvrE and HopBJ1 ultimately lead to the same physiological outcomes during infection but, thus far, little is known about processes targeted by AvrE.

What advantages HopBJ1 provides to $\mathrm{CC} 1557$ in nature remains unknown, because it is currently unclear whether this strain is capable of causing disease in any organism outside of the lab environment or syringe infiltration. Indeed, both CC1557 (snow) and CC1466 (asymptomatic plants) were originally isolated in order to characterize environmental diversity of $P$. syringae strains outside of crop disease. It is certainly possible that $\mathrm{CC} 1557$ and related strains persist at low levels across a range of plant hosts and habitats, using a T3SS and toxins to survive epiphytically but never reaching sufficiently large 
population sizes to cause disease. It is also possible that CC1557 is naturally pathogenic within a suite of understudied plant hosts or only under specific environmental conditions. However, given the disease symptoms measured under laboratory conditions, it is clear that $\mathrm{CC} 1557$ has the potential to cause rapid cell death in plant leaves relative to other $P$. syringae strains and that a significant amount of this activity is dependent on HopBJ1. These results suggest an intriguing possibility that the disease ecology of CC1557 is fundamentally different (for instance, more necrotrophic) than other commonly studied $P$. syringae strains.

Herein, we have analyzed the genomes of two $P$. syringae strains isolated from environmental sources. These genomes contain a paucity of known T3E, even though virulence of CC1557 on $N$. benthamiana is dependent on a functioning T3SS. That these strains have also acquired an NRP pathway

Table 3. Strains and plasmids ${ }^{\mathrm{a}}$

\begin{tabular}{|c|c|c|c|}
\hline Name & Description & Antibiotics & Reference \\
\hline \multicolumn{4}{|l|}{ Strain } \\
\hline CC1466 & Pseudomonas syringae CC1466 & $\ldots$ & Morris et al. 2008 \\
\hline $\mathrm{CC} 1557$ & P. syringae $\mathrm{CC} 1557$ & $\ldots$ & Morris et al. 2008 \\
\hline CC1583 & P. syringae $\mathrm{CC} 1583$ & $\ldots$ & Morris et al. 2008 \\
\hline DBL627 & rifR version of $P$. syringae $\mathrm{CC} 1557$ & $\ldots$ & This study \\
\hline DBL704 & DBL627 with pDAB334, CC1557 rif ${ }^{\mathrm{R}}$ mutant with EV & $\ldots$ & This study \\
\hline DBL690 & DBL627 with pDBL048 integrated, hopBJI deletion construct integrated into & & \\
\hline & $\mathrm{CC} 1557$ rif $^{\mathrm{R}^{\mathrm{T}}}$ & $\ldots$ & This study \\
\hline DBL692 & DBL627 with pDBL051 integrated, $h r c C$ deletion construct integrated into & & \\
\hline & $\mathrm{CC} 1557 \operatorname{rif}^{\mathrm{R}}$ & $\ldots$ & This study \\
\hline DBL698 & DBL627 hrcC mutant & $\ldots$ & This study \\
\hline DBL696 & DBL627 hopBJ1 mutant & $\ldots$ & This study \\
\hline DBL704 & DBL627 with EV pDAB334, CC1557 rif $^{\mathrm{R}}$ with EV & $\ldots$ & This study \\
\hline DBL706 & DBL696 del with pDAB334, hopBJI deletion with EV & $\ldots$ & This study \\
\hline DBL705 & DBL696 del with pDBL052, hopBJ1 deletion with hopBJI & $\ldots$ & This study \\
\hline DBL708 & DBL698 with pDAB334, CC1557 rif $^{\mathrm{R}} h r c C$ - with EV & $\ldots$ & This study \\
\hline DBL707 & DBL698 with pDBL052, CC1557 rif ${ }^{\mathrm{R}} h r c C$ - with $h o p B J 1$ & $\ldots$ & This study \\
\hline DBL604 & P. syringae pv. tomato DC3000 with pDBL061 & $\ldots$ & This study \\
\hline DBL888 & DC3000 hrcC mutant with hopBJ1 (no promoter) pDBL061 & $\ldots$ & This study \\
\hline MTN3101 & GV3101 with pMTN3100; 35S HopBJ1:YFP & $\ldots$ & This study \\
\hline MTN3131 & GV3101 with pMTN3100; 35S HopBJ1:YFP & $\ldots$ & This study \\
\hline MTN3132 & GV3101 with pMTN3100; 35S HopBJ1:YFP & $\ldots$ & This study \\
\hline MTN2890 & DC3000 with pDBL052; HopBJ1 in pJC531 & $\ldots$ & This study \\
\hline MTN3151 & DC3000 with pMTN3149; HopBJ1 C174S in pJC531 & $\ldots$ & This study \\
\hline MTN3152 & DC3000 with pMTN3150; HopBJ1 H192A in pJC531 & $\ldots$ & This study \\
\hline MTN3155 & DBL627 with pMTN3149; HopBJ1 C174S in pJC531 & $\ldots$ & This study \\
\hline MTN3156 & DBL627 with pMTN3150; HopBJ1 H192A in pJC531 & $\ldots$ & This study \\
\hline MTN3240 & DC3000-D28E with pMTN3224; HSP18-2 UTR in pJC532 & $\ldots$ & This study \\
\hline MTN3241 & DC3000-D28E with pMTN3225; HopBJ1 in pJC532 & $\ldots$ & This study \\
\hline MTN3242 & DC3000-D28E with pMTN3226; HopBJ1 C174S in pJC532 & $\ldots$ & This study \\
\hline MTN3243 & DC3000-D28E with pMTN3227; HopBJ1 H192A in pJC532 & $\ldots$ & This study \\
\hline \multicolumn{4}{|l|}{ Plasmids } \\
\hline pMTN1907 & Gateway destination vector for making clean deletions in $P$. syringae & $\mathrm{Tet}^{\mathrm{R}}, \mathrm{Kan}^{\mathrm{R}}, \mathrm{Cam}^{\mathrm{R}}, \mathrm{Suc}^{\mathrm{S}}$ & Baltrus et al. 2012 \\
\hline pDONR207 & Gateway entry vector from Invitrogen & $\mathrm{Gent}^{\mathrm{R}}, \mathrm{Cam}^{\mathrm{R}}$ & Invitrogen \\
\hline pMTN1970 & $3^{\prime}$ UTR of Arabidopsis HSP18.2 in pDONR207 & Gent $^{R}$ & Baltrus et al. 2012 \\
\hline pJC531 & & & \\
\hline \multirow{2}{*}{ pJC532 } & $\begin{array}{l}\text { C-terminal HA tag } \\
\text { Gateway destination vector based off of pBBRMCS1 no promoter; }\end{array}$ & $\mathrm{Kan}^{\mathrm{R}}, \mathrm{Cam}^{\mathrm{R}}$ & Chang et al. 2005 \\
\hline & C-terminal $\Delta$ AvrRpt2 & $\mathrm{Kan}^{\mathrm{R}}, \mathrm{Cam}^{\mathrm{R}}$ & Chang et al. 2005 \\
\hline pDAB334 & MTN1970-2 EV construct in pJC531 & $\operatorname{Kan}^{\mathrm{R}}$ & Baltrus et al. 2012 \\
\hline pDBL040 & hopBJ1 cloned with promoter, no stop codon in pDONR207 & Gent $^{R}$ & This study \\
\hline pMTN3111 & hopBJ1 C174S with promoter, no stop codon in pDONR207 & Gent $^{\mathrm{R}}$ & This study \\
\hline pMTN3112 & hopBJ1 H192A with promoter, no stop codon in pDONR207 & Gent $^{\mathrm{R}}$ & This study \\
\hline pDBL052 & hopBJ1 cloned with promoter, no stop codon in pJC531 & $\mathrm{Kan}^{\mathrm{R}}$ & This study \\
\hline pMTN3149 & hopBJ1 C174S cloned with promoter, no stop codon in pJC531 & $\mathrm{Kan}^{\mathrm{R}}$ & This study \\
\hline pMTN3150 & hopBJ1 H192A cloned with promoter, no stop codon in pJC531 & $\mathrm{Kan}^{\mathrm{R}}$ & This study \\
\hline pDBL042 & hopBJ1 deletion construct in pDONR207 & Gent $^{R}$ & This study \\
\hline pDBL048 & hopBJI deletion construct in MTN1907 & $\mathrm{Tet}^{\mathrm{R}}$ & This study \\
\hline pDBL012 & hopBJ1 cloned without promoter into pDONR207, no stop & Gent $^{R}$ & This study \\
\hline pMTN3113 & hopBJ1 C174S ORF, no stop codon in pDONR207 & Gent $^{R}$ & This study \\
\hline pMTN3114 & hopBJ1 H192A ORF, no stop codon in pDONR207 & Gent $^{R}$ & This study \\
\hline pDBL061 & hopBJ1 cloned without promoter into pBAV187 & $\mathrm{Tet}^{\mathrm{R}}$ & This study \\
\hline pMTN3224 & HSP18.2 UTR in pJC532 & $\mathrm{Kan}^{\mathrm{R}}$ & This study \\
\hline pMTN3225 & hopBJI cloned with promoter, no stop codon in $\mathrm{pJC} 532$ & $\operatorname{Kan}^{\mathrm{R}}$ & This study \\
\hline pMTN3226 & hopBJ1 C174S cloned with promoter, no stop codon in pJC532 & $\mathrm{Kan}^{\mathrm{R}}$ & This study \\
\hline pMTN3227 & hopBJ1 H192A cloned with promoter, no stop codon in pJC532 & $\operatorname{Kan}^{\mathrm{R}}$ & This study \\
\hline pDBL045 & CC1557 hrcC deletion construct in pDONR207 & Gent $^{\mathrm{R}}$ & This study \\
\hline pDBL051 & CC1557 hrcC deletion construct in MTN1907 & $\mathrm{Tet}^{\mathrm{R}}$ & This study \\
\hline pGWB641 & Gateway destination vector; $35 \mathrm{~S}$ promoter, C-terminal YFP & $\mathrm{Spec}^{\mathrm{R}}, \mathrm{Cam}^{\mathrm{R}}$ & Nakamura et al. 2010 \\
\hline pMTN3100 & hopBJ1 ORF in pGWB641 & $\operatorname{Spec}^{\mathrm{R}}$ & This study \\
\hline pMTN3119 & hopBJ1 C174S ORF in pGWB641 & $\mathrm{Spec}^{\mathrm{R}}$ & This study \\
\hline pMTN3120 & hopBJ1 H192A ORF in pGWB641 & Spec $^{R}$ & This study \\
\hline
\end{tabular}

${ }^{a}$ rif $^{\mathrm{R}}, \mathrm{Tet}^{\mathrm{R}}, \mathrm{Kan}^{\mathrm{R}}, \mathrm{Cam}^{\mathrm{R}}, \mathrm{Gent}^{\mathrm{R}}$, and $\mathrm{Spec}^{\mathrm{R}}$ indicate resistant to rifampicin tetracycline, kanamycin, chloramphenicol, gentamycin, and spectinomycin; Suc ${ }^{\mathrm{S}}$ indicates sensitive to sucrose; $\mathrm{EV}=$ empty vector, $\mathrm{UTR}=$ untranslated region, and $\mathrm{ORF}=$ open reading frame. 
similar to syringomycin represents an independent evolutionary example that supports a negative correlation between NRP pathways and T3E repertoires. Furthermore, this data set highlights how genomic scans can lead to insights into pathogenesis while also demonstrating the power of genetic diversity to uncover genomewide changes in virulence gene architecture.

\section{MATERIALS AND METHODS}

Plasmids, bacterial isolates, and growth conditions.

All bacterial strains and plasmids used or created are listed in Table 3. Typically, P. syringae and Agrobacterium isolates were grown at $27^{\circ} \mathrm{C}$ on King's B (KB) media using $50 \mu \mathrm{g} / \mathrm{ml}$ rifampicin at $50 \mu \mathrm{g} / \mathrm{ml}$. When necessary, cultures of $P$. syringae, Agrobacterium spp., and E. coli were supplemented with antibiotics or sugars in the following concentrations: tetracycline at $10 \mu \mathrm{g} / \mathrm{ml}$, kanamycin at $50 \mu \mathrm{g} / \mathrm{ml}$, spectinomycin at 50 $\mu \mathrm{g} / \mathrm{ml}$, gentamycin at $25 \mu \mathrm{g} / \mathrm{ml}(50 \mu \mathrm{g} / \mathrm{ml}$ for Agrobacterium spp.), and $5 \%$ sucrose.

All clones were created by first amplifying target sequences using $P f x$ polymerase (Invitrogen, Waltham, MA, U.S.A.), followed by recombination of these fragments into the entry vector pDONR207 using BP clonase (Invitrogen). Site-directed mutagenesis was performed using SLIM amplification (Chiu et al. 2004). All ORF (without an hrp-box promoter) and gene (including the promoter) sequences were confirmed by Sanger sequencing of these pDONR207 clones. Clones in entry vectors were recombined into destination vectors of interest using LR clonase (Invitrogen).

\section{Genome sequences and searches.}

Draft genome assemblies for CC1466, CC1557, and CC1583 are publicly available through GenBank (accession numbers AVEM00000000, AVEH00000000, and AVEG00000000, respectively) (Baltrus et al. 2014). One PacBio SMRT cell yielded 37,509 reads for a total of 268,122,626 nucleotides after filtering for quality. To piece together the complete genome sequence for CC1557, PacBio reads were first assembled using the HGAP software (Chin et al. 2013), yielding two contigs total with no scaffolding gaps. Contigs from the previous assembly (using only 100-bp Illumina paired-end reads) (Baltrus et al. 2014) were then overlaid onto this HGAP assembly. When there was coverage by contigs from the Illumina assembly, the previous assembly sequence was chosen as the final sequence but, when there was no coverage, the PacBio assembly was chosen. In cases where multiple divergent Illumina contigs assembled to the same region in the HGAP assembly, the Illumina sequence which matched the PacBio assembly was chosen. Because the second contig from the HGAP assembly possesses numerous plasmid-related genes, we are confident that this does actually represent a plasmid present within this strain. Both the chromosome and plasmid were annotated using PGAAP (Angiuoli et al. 2008).

T3E and NRP pathways were identified as per Baltrus and associates (2011). Briefly, draft genome assemblies were queried using tBLASTn, with queries consisting of protein sequences for known T3E families or for key loci within toxin pathways. Each BLAST hit was validated by hand for copy number, identity, and completeness. All BLAST results were visually inspected to make sure that each sequence displayed high similarity to only one region in the assembly, that this similar region was not part of a larger ORF, and that the length of this region was greater than $40 \%$ of the length of the original query sequence.

The Phyre 2 server was used to carry out protein structure similarity searches (Kelley and Sternberg 2009). In brief, this server performs an automated search of both the protein se- quence and predicted folds for proteins of interest (in this case, HopBJ1) across multiple databases. Within this pipeline, iterative searches such as PSI-Blast enable identification of distantly similar sequence matches. Once a profile is constructed for the sequence of interest, it is compared against a database of known protein structures and returns the best matches as a prediction of protein folds within the query.

\section{Generation of bacterial mutants.}

Bacterial mutants were generated as per Baltrus and associates (2012). Regions ( $>500 \mathrm{bp}$ ) upstream and downstream of the target genes were polymerase chain reaction (PCR) amplified separately and then combined into one fragment by overlap extension PCR. The bridge PCR amplicon was then cloned into pDONR207, and further moved into pMTN1907 using LR clonase. Once mated into $P$. syringae, single recombination of a homologous region upstream or downstream of the target region and subsequent selection on sucrose allowed for screening of clean deletions. Mutants were confirmed by phenotyping for sucrose resistance, tetracycline sensitivity, PCR amplification of the deletion, and failure of PCR to amplify regions within the deletion.

\section{Negative correlation between syringomycin-like toxin pathways and T3E.}

We calculated statistical significance for negative correlation between toxin pathways and effectors using data on the number of full-length T3E per strain from Baltrus and associates (2011) as well as data from Table 1. To minimize bias due to phylogenetic relationships, we chose more than five diverse strains from MLSA groups 1, 2, and 3 as well as CC1557 and CC1583. We then performed a Wilcoxon rank sum test to compare the number of full-length effectors between genomes which either contain or lack the genetic capacity for production of a syringomycin-like toxin.

\section{In planta growth curves, cell-death assays, and translocation tests.}

$N$. benthamiana plants were grown for 4 to 6 weeks on a long-day cycle (16 h of light and $8 \mathrm{~h}$ of darkness). Plants were removed from the growth chamber and allowed to acclimate to ambient laboratory conditions for a period of 3 to 5 days prior to infiltration (for experiments taking place at the University of Arizona). Bacteria were cultured overnight on $\mathrm{KB}$ amended with proper antibiotics. Bacterial cells were washed $1 \times$ and resuspended to an optical density at $600 \mathrm{~nm}\left(\mathrm{OD}_{600}\right)$ of 0.002 or 0.0002 in sterile $10 \mathrm{mM} \mathrm{MgCl}$, yielding a final inoculum density of approximately $1 \times 10^{5}$ (for experiments taking place at the University of North Carolina) or $1 \times 10^{6} \mathrm{CFU} / \mathrm{ml}$ (for experiments taking place at the University of Arizona). Cell suspensions were then syringe infiltrated into the abaxial side of $N$. benthamiana leaves. Populations were recovered after two (for experiments taking place at the University of North Carolina) or three (for experiments taking place at the University of Arizona) days of growth using a cork borer, unless otherwise noted. Leaf disks were disrupted using glass beads and a bead-beater device and populations were enumerated by dilution plating onto $\mathrm{KB}$ amended with appropriate antibiotics. Arabidopsis growth assays were performed similarly, with the exception that plants were grown in walk-in rooms maintained on a short-day cycle $\left(9 \mathrm{~h}\right.$ of light at $21^{\circ} \mathrm{C}$ and $15 \mathrm{~h}$ of darkness at $\left.18^{\circ} \mathrm{C}\right)$.

Cell death induced by HopBJ1 was assayed after transient expression of 35S-driven HopBJ1:YFP in $N$. benthamiana. Bacteria were grown to saturation overnight in $2 \times$ yeast tryptone media and then diluted to an $\mathrm{OD}_{600}$ of 0.1 . Cultures were syringe injected into fully expanded leaves of 5- to 6-week-old 
plants and cell death was visualized $24 \mathrm{~h}$ postinoculation. Accumulation of wild-type and mutant HopBJ1 was verified with standard Western blotting of lysates from injected leaf cores using anti-GFP (Roche, Penzeburg, Germany).

HopBJ1 was tested for the ability to secrete the active C-terminal fragment of AvrRpt 2 to cause a hypersensitive response (HR) in Arabidopsis accession Col-0 (Guttman et al. 2002). For this test, promoterless HopBJl was cloned into plasmid pBAV178 (Vinatzer et al. 2006) so that expression in strain DC3000 D28E was dependent on a tet promoter. This construct was also placed into an $h r c C$ - mutant of DC3000 (Baltrus et al. 2012), in order to test the requirement of a functioning T3SS for tissue collapse. All HR tests were performed on approximately 5-week-old plants and utilized a bacterial density of $\mathrm{OD}_{600}=0.05$. Tissue collapse was measured at either 24 or $48 \mathrm{~h}$ postinfection and confirmed by at least four independent tests. For translocation of HopBJ1 and HopBJ1 sitedirected mutants, similar assays were done with native promoter clones in pJC532 delivered from the D28E effector-deleted strain of DC3000. (Chang et al. 2005).

\section{Phylogenetic methods.}

Phylogenetic analyses were performed on concatenated MLSA loci as described previously using concatenated fragments from seven MLSA genes across a diverse array of isolates from $P$. syringae (Baltrus et al. 2011) as well as AvrE and HrpW protein sequences. MrBayes was used to perform Bayesian phylogenetic analyses with flat priors, a burn-in period of 250,000 generations, and convergence after 1,000,000 total generations (Ronquist et al. 2012).

\section{ACKNOWLEDGMENTS}

We thank the University of Delaware Sequencing Core Facility for technical help with PacBio sequences; C. Morris, INRA, France for kindly providing access to strains described in this article; E. H. Chung for reagents; and J. Dangl for experimental reagents and positive encouragement. Funding was provided by startup funds to D. A. Baltrus from the University of Arizona.

\section{LITERATURE CITED}

Angiuoli, S. V., Gussman, A., Klimke, W., Cochrane, G., Field, D., Garrity, G., Kodira, C. D., Kyrpides, N., Madupu, R., Markowitz, V., Tatusova, T., Thomson, N., and White, O. 2008. Toward an online repository of standard operating procedures (SOPs) for (meta)genomic annotation. OMICS 12:137-141.

Araki, H., Tian, D., Goss, E. M., Jakob, K., Halldorsdottir, S. S., Kreitman, M., and Bergelson, J. 2006. Presence/absence polymorphism for alternative pathogenicity islands in Pseudomonas viridiflava, a pathogen of Arabidopsis. Proc. Natl. Acad. Sci. U.S.A. 103:5887-5892.

Badel, J. L., Shimizu, R., Oh, H.-S., and Collmer, A. 2006. A Pseudomonas syringae pv. tomato avrE1/hopM1 mutant is severely reduced in growth and lesion formation in tomato. Mol. Plant-Microbe Interact. 19:99-111.

Baltrus, D. A., Nishimura, M. T., Romanchuk, A., Chang, J. H., Mukhtar, M. S., Cherkis, K., Roach, J., Grant, S. R., Jones, C. D., and Dangl, J. L. 2011. Dynamic evolution of pathogenicity revealed by sequencing and comparative genomics of 19 Pseudomonas syringae isolates. PLoS Pathog. 7:e1002132. Published online.

Baltrus, D. A., Nishimura, M. T., Dougherty, K. M., Biswas, S., Mukhtar, M. S., Vicente, J., Holub, E. B., and Dangl, J. L. 2012. The molecular basis of host specialization in bean pathovars of Pseudomonas syringae. Mol. Plant-Microbe Interact. 25:877-888.

Baltrus, D. A., Dougherty, K., Beckstrom-Sternberg, S. M., BeckstromSternberg, J. S., and Foster, J. T. 2013. Incongruence between MLSA and whole genome phylogenies: Pseudomonas syringae as a cautionary tale. Mol. Plant Pathol. 15:461-465.

Baltrus, D. A., Yourstone, S., Lind, A., Guilbaud, C., Sands, D. C., Jones, C. D., Morris, C. E., and Dangl, J. L. 2014. Draft genomes for a phylogenetically diverse suite of Pseudomonas syringae strains from multiple source populations. Genome Announce. 23:e01195-13. doi:10.1128/
genomeA.01195-13.

Bartoli, C., Berge, O., Monteil, C. L., Guilbaud, C., Balestra, G. M., Varvaro, L., Jones, C., Dangl, J. L., Sands, D. C., and Morris, C. E. 2014. The Pseudomonas viridiflava phylogroups in the $P$. syringae species complex are characterized by genetic variability and phenotypic plasticity of pathogenicity-related traits. Environ. Microbiol doi:10.1111/1462-2920.12433. Published online.

Bender, C. L., Alarcón-Chaidez, F., and Gross, D. C. 1999. Pseudomonas syringae phytotoxins: Mode of action, regulation, and biosynthesis by peptide and polyketide synthetases. Microbiol. Mol. Biol. Rev. 63:266292.

Buetow, L., Flatau, G., Chiu, K., Boquet, P., and Ghosh, P. 2001. Structure of the Rho-activating domain of Escherichia coli cytotoxic necrotizing factor 1. Nat. Struct. Biol. 8:584-588.

Chang, J. H., Urbach, J. M., Law, T. F., Arnold, L. W., Hu, A., Gombar, S., Grant, S. R., and Dangl, J. L. 2005. A high-throughput, near saturating screen for type III effector genes from Pseudomonas syringae. Proc. Natl. Acad. Sci. U.S.A. 102:2549-2554.

Chin, C.-S., Alexander, D. H., Marks, P., Klammer, A. A., Drake, J., Heiner, C., Clum, A., Copeland, A., Huddleston, J., Eichler, E. E., Turner, S. W., and Korlach, J. 2013. Nonhybrid, finished microbial genome assemblies from long-read SMRT sequencing data. Nat. Methods 10:563-569.

Chiu, J., March, P. E., Lee, R., and Tillett, D. 2004. Site-directed, Ligaseindependent mutagenesis (SLIM): A single-tube methodology approaching $100 \%$ efficiency in $4 \mathrm{~h}$. Nucleic Acids Res. 32:e174.

Clarke, C. R., Cai, R., Studholme, D. J., Guttman, D. S., and Vinatzer, B. A. 2010. Pseudomonas syringae strains naturally lacking the classical P. syringae hrp/hrc locus are common leaf colonizers equipped with an atypical type III secretion system. Mol. Plant-Microbe Interact. 23:198210.

Cui, H., Wang, Y., Xue, L., Chu, J., Yan, C., Fu, J., Chen, M., Innes, R. W., and Zhou, J.-M. 2010. Pseudomonas syringae effector protein AvrB perturbs Arabidopsis hormone signaling by activating MAP kinase 4. Cell Host Microbe 7:164-175.

Cunnac, S., Chakravarthy, S., Kvitko, B. H., Russell, A. B., Martin, G. B., and Collmer, A. 2011. Genetic disassembly and combinatorial reassembly identify a minimal functional repertoire of type III effectors in Pseudomonas syringae. Proc. Natl. Acad. Sci. U.S.A. 108:2975-2980.

Feil, H., Feil, W. S., Chain, P., Larimer, F., DiBartolo, G., Copeland, A., Lykidis, A., Trong, S., Nolan, M., Goltsman, E., Thiel, J., Malfatti, S. Loper, J. E., Lapidus, A., Detter, J. C., Land, M., Richardson, P. M., Kyrpides, N. C., Ivanova, N., and Lindow, S. E. 2005. Comparison of the complete genome sequences of Pseudomonas syringae pv. syringae B728a and pv. tomato DC3000. Proc. Natl. Acad. Sci. U.S.A. 102:11064-11069.

Guttman, D. S., Vinatzer, B. A., Sarkar, S. F., Ranall, M. V., Kettler, G., and Greenberg, J. T. 2002. A functional screen for the type III (Hrp) secretome of the plant pathogen Pseudomonas syringae. Science 295:1722-1726.

Henty-Ridilla, J. L., Shimono, M., Li, J., Chang, J. H., Day, B., and Staiger, C. J. 2013. The plant actin cytoskeleton responds to signals from microbe-associated molecular patterns. PLoS Pathog. 9:e1003290. Published online.

Hirano, S., and Upper, C. 2000. Bacteria in the leaf ecosystem with emphasis on Pseudomonas syringae-a pathogen, ice nucleus, and epiphyte. Microbiol. Mol. Biol. Rev. 64:624.

Hwang, M. S. H., Morgan, R. L., Sarkar, S. F., Wang, P. W., and Guttman, D. S. 2005. Phylogenetic characterization of virulence and resistance phenotypes of Pseudomonas syringae. Appl. Environ. Microbiol. 71:5182-5191.

Jiang, S., Yao, J., Ma, K.-W., Zhou, H., Song, J., He, S. Y., and Ma, W. 2013. Bacterial effector activates jasmonate signaling by directly targeting JAZ transcriptional repressors. PLoS Pathog. 9:e1003715. Published online.

Kelley, L. A., and Sternberg, M. J. E. 2009. Protein structure prediction on the Web: A case study using the Phyre server. Nat. Protocols 4:363-371.

Kobayashi, D. Y., Tamaki, S. J., and Keen, N. T. 1989. Cloned avirulence genes from the tomato pathogen Pseudomonas syringae pv. tomato confer cultivar specificity on soybean. Proc. Natl. Acad. Sci. U.S.A. 86:157-161.

Kvitko, B. H., Park, D. H., Velásquez, A. C., Wei, C.-F., Russell, A. B., Martin, G. B., Schneider, D. J., and Collmer, A. 2009. Deletions in the repertoire of Pseudomonas syringae pv. tomato DC3000 type III secretion effector genes reveal functional overlap among effectors. PLoS Pathog. 5:e1000388. Published online.

Lee, A. H., Hurley, B., Felsensteiner, C., Yea, C., Ckurshumova, W., Bartetzko, V., Wang, P. W., Quach, V., Lewis, J. D., Liu, Y. C., Bornke, F., Angers, S., Wilde, A., Guttman, D. S., and Desveaux, D. 2012. A bacterial acetyltransferase destroys plant microtubule networks and 
blocks secretion. PLoS Pathog. 8:e1002523. Published online.

Lemonnier, M., Landraud, L., and Lemichez, E. 2007. Rho GTPase-activating bacterial toxins: From bacterial virulence regulation to eukaryotic cell biology. FEMS (Fed. Eur. Microbiol. Soc.) Microbiol. Rev. 31:515-534.

Lindeberg, M., Cunnac, S., and Collmer, A. 2012. Pseudomonas syringae type III effector repertoires: Last words in endless arguments. Trends Microbiol. 20:199-208.

Melotto, M., Underwood, W., Koczan, J., Nomura, K., and He, S. Y. 2006. Plant stomata function in innate immunity against bacterial invasion. Cell 126:969-980.

Morris, C. E., Sands, D. C., Vinatzer, B. A., Glaux, C., Guilbaud, C., Buffière, A., Yan, S., Dominguez, H., and Thompson, B. M. 2008. The life history of the plant pathogen Pseudomonas syringae is linked to the water cycle. ISME J. 2:321-334.

Mucha, E., Fricke, I., Schaefer, A. Wittinghofer, A., and Berken, A. 2011. Rho proteins of plants-Functional cycle and regulation of cytoskeletal dynamics. Eur. J. Cell Biol. 90:934-943.

Mucyn, T. S., Yourstone, S., Lind, A. L., Biswas, S., Nishimura, M. T., Baltrus, D. A., Cumbie, J. S., Chang, J. H., Jones, C. D., Dangl, J. L., and Grant, S. R. 2014. "Variable suites of non-effector genes are coregulated in the type III secretion virulence regulons across the Pseudomonas syringae phylogeny”. PLoS Pathog. 10:e1003807. doi:10.1371/ journal.ppat.1003807. Published online.

Nakamura, S., Mano, S., Tanaka, Y., Ohnishi, M., Nakamori, C., Araki, M., Niwa, T., Nishimura, M., Kaminaka, H., Nakagawa, T., Sato, Y., and Ishiguro, S. 2010. Gateway binary vectors with the bialaphos resistance gene, bar, as a selection marker for plant transformation. Biosci. Biotechnol. Biochem. 74:1315-1319.

O'Brien, H. E., Thakur, S., and Guttman, D. S. 2011. Evolution of plant pathogenesis in Pseudomonas syringae: A genomics perspective. Annu.
Rev. Phytopathol. 49:269-289.

Quigley, N. B., and Gross, D. C. 1994. Syringomycin production among strains of Pseudomonas syringae pv. syringae: Conservation of the syrB and $s y r D$ genes and activation of phytotoxin production by plant signal molecules. Mol. Plant-Microbe Interact. 7:78-90.

Ramos, C., Matas, I. M., Bardaji, L., Aragón, I. M., and Murillo, J. 2012. Pseudomonas savastanoi pv. savastanoi: Some like it knot. Mol. Plant Pathol. 13:998-1009.

Ronquist, F., Teslenko, M., van der Mark, P., Ayres, D. L., Darling, A., Hohna, S., Larget, B., Liu, L., Suchard, M. A., and Huelsenbeck, J. P. 2012. MrBayes 3.2: Efficient Bayesian phylogenetic inference and model choice across a large model space. Syst. Biol. 61:539-542.

Sarkar, S. F., and Guttman, D. S. 2004. Evolution of the core genome of Pseudomonas syringae, a highly clonal, endemic plant pathogen. Appl. Environ. Microbiol. 70:1999-2012.

Schellenberg, B., Ramel, C., and Dudler, R. 2010. Pseudomonas syringae virulence factor syringolin A counteracts stomatal immunity by proteasome inhibition. Mol. Plant-Microbe Interact. 23:1287-1293.

Studholme, D. J. 2011. Application of high-throughput genome sequencing to intrapathovar variation in Pseudomonas syringae. Mol. Plant Pathol. 12:829-838.

Vinatzer, B. A., Teitzel, G. M., Lee, M.-W., Jelenska, J., Hotton, S., Fairfax, K., Jenrette, J., and Greenberg, J. T. 2006. The type III effector repertoire of Pseudomonas syringae pv. syringae B728a and its role in survival and disease on host and non-host plants. Mol. Microbiol. 62:26-44.

\section{AUTHOR-RECOMMENDED INTERNET RESOURCE}

Phyre2 server, http://www.sbg.bio.ic.ac.uk/phyre2/html/page.cgi?id=index 